\title{
A case of unsuspected chronic lithium toxicity presenting with neurological symptoms.
}

\author{
Umme Kulsum Chy ${ }^{1}$, Amina Sultana ${ }^{2}$, Mohammad Omar Faruq ${ }^{3 *}$ \\ DOI: https://doi.org/10.3329/bccj.v9i1.53058
}

\begin{abstract}
:
Lithium Carbonate is the first line treatment for bipolar depressive disorder. It also has beneficial effects on prophylactic use in Acute manic episodes to prevent recurrence and reduces rate of suicide associated with affective disorder. Low therapeutic index of Lithium (0.8-1.2meq/l) mandates regular and frequent measurement of Serum lithium level in patients taking this drug. Here we present a 59 years Indian male who is a known diabetic, chronic alcoholic and a case of bipolar disorder. He was on Lithium tablet off and on for last 4 years and was also on oral antidiabetic agent. He was admitted in ICU through emergency with complaints of altered level of consciousness for 2 days with $\mathrm{H} / \mathrm{O}$ recurrent hypoglycemia associated with nausea, reduced food intake, vertigo, tremor of all four limbs with weakness of both lower limbs for approximately last 2 months. Admission serum lithium level was 2.24meq/l. Hemodialysis was started. Neurological symptoms improved and serum Lithium level returned to normal (0.79meq/l) after 2 session of hemodialysis.
\end{abstract}

Key words: Lithium, Bipolar Disorder, Parkinson's disease, Hemodialysis.

\section{Introduction:}

Among antipsychotics for bipolar disorder, lithium carbonate is the first line treatment of choice. It's also used for acute manic episodes and it reduces rate of suicide associated with affective disorder. Narrow therapeutic index of lithium (0.6-1.2 meq/l) requires regular monitoring for optimum prophylaxis and avoidance of toxic effects. Variable elimination half-life of lithium ranging from 18 hours to 60 hours depending on age and renal function of different subjects results in poor prediction of toxicity ${ }^{1}$. Mechanism of action is supposed to be through direct interaction between lithium ion and cellular enzyme influencing secondary signaling pathway of dopamine, serotonin and norepinephrine. Factors influencing accumulation of lithium and thus resulting in toxicity includes NSAIDs, diuretics, angiotensin converting enzyme inhibitors, low output heart failure, advanced age and conditions that result in sodium intake such GI loss. Grades of side effects are classified as mild $(1.5-2.5 \mathrm{meq} / 1)$, moderate $(2.5-3.5 \mathrm{meq} / \mathrm{l})$ and severe $(>3.5 \mathrm{meq} / \mathrm{l})$ according to clinical feature. Presentations are categorized as Acute, Acute on chronic and Chronic depending on duration and doses of drug intake $e^{1,2}$. Acute lithium overdose usually presents more with GI toxicity and less neurotoxicity ${ }^{1}$. In acute on chronic toxicity clinical manifestations are both of GI and neurologic symptoms.

1. Junior Consultant, GICU, United Hospital Ltd, Gulshan, Dhaka 1212

2. Associate consultant, GICU and Covid ICU, United Hospital, Gulshan Dhaka 1212.

3. Chief Consultant, GICU and Emergency, United Hospital, Gulshan, Dhaka 1212

*Corresponding Author:

Professor Mohammad Omar Faruq

e-mail: faruqmo@yahoo.com
Baseline investigation before initiation of lithium should include renal function, serum electrolytes including calcium and thyroid profile, as chronic therapy may ended up with renal impairment with nephrogenic diabetes insipidus, hypothyroidism, hyperparathyroidism, hypercalcemia etc ${ }^{3}$.We present 59 year old Indian male who has been on lithium irregularly for bipolar disorder and who presented with altered level of consciousness with hallucination with complaints of nausea, anorexia, fine tremor of upper limbs with weakness of lower limbs. He visited consultants of different specialties for his complaints for about 2 months before admission to our ICU.

\section{Case Report}

59 years Indian, diabetic (on oral antidiabetic agent), alcoholic divorced gentleman admitted to ICU of a tertiary care hospital in Dhaka on $24^{\text {th }}$ January 2021 with complaints of nausea with reduced intake of food for about last 10 days associated with sleeplessness, altered level of consciousness along with irrelevant talking for 3-4 days with weakness of both lower limbs resulting in inability to walk. Patient has been residing in Bangladesh for about 5 years without any family member or close relatives. As such it became difficult to obtain relevant history. According to his colleagues who brought him to emergency room, he had been consulting with different physician in different hospitals with the complaints of tremor of hands, vertigo and weakness of both lower limbs. Several investigations including NCV of both lower limb were advised and treatment given as a suspected case of Parkinsonism. Only one prescription confirmed drug history of having Lithium $600 \mathrm{mg} 2 \mathrm{tab}$ daily. On further enquiry it became known that he had been on lithium tablet for about last 4 years prescribed by his physician in India but his Lithium therapy was unsupervised. It was suspected that he was taking Lithium irregularly. With reduced level of consciousness patient was taken to a local hospital on day prior to visit to our hospital emergency. Here he was found to be hypoglycemic ( blood glucose $2.3 \mathrm{mmol} / \mathrm{l}$ ) and treated 
accordingly. Patient was advised for admission but he refused. The following day patient was brought to our hospital because of further deterioration of his status. Initial investigation including serum lithium level revealed high lithium level $(2.7 \mathrm{meq} / \mathrm{l})$ and raised serum creatinine $(1.43 \mathrm{mg} / \mathrm{dl})$ with reduced glomerular filtration rate. As patient was hypotensive despite fluid challenge, he was admitted in to ICU. On admission patient was restless with irrelevant and incoherent speech, disoriented to time, place and person. He was continuously talking to some imaginary personand it was difficult to differentiate whether it was auditory hallucination or visual. On physical examination, patient was found to be dehydrated, blood pressure was $80 / 50 \mathrm{mmHg}$. On Neurological assessment of patient there was cognitive impairment and cerebellar sign couldn't be evaluated because of patient's restlessness. Muscle power was normal, planter were B/L flexor. Initial blood gas was absolutely fine. Blood lithium level was $2.24 \mathrm{meq} / 1$ with renal impairment (serum creatinine $1.42 \mathrm{mg} / \mathrm{dl}$ ). Inotrope started after adequate fluid challenge as blood pressure didn't improve. CSF examination was unremarkable and CT scan of brain was normal. Adequate hydration ensured and subsequently two course of hemodiafiltration were given on day three and day four after admission. Serial measurement of serum lithium level done daily and after 5 days of ICU admission it was $0.79 \mathrm{meq} / 1$ with significant improvement of neurological status. MRI brain also done which was reported to be normal. From ICU he was shifted out under care of a Psychiatrist.

Continuation of drug along with poor hydration and poor oral intake resulting into reduced renal function leads to lithium toxicity with acute psychosis in this patient.

\section{Discussion}

In our patient continuation of Lithium along with poor hydration and poor oral intake resulted into reduced renal function and it ultimately led to lithium toxicity with acute psychosis.

Mechanism of lithium toxicity is not yet clearly understood. Lithium toxicity can present with mild symptoms like nausea, vomiting, diarrhea or even can be fatal with neurotoxicity, renal impairment or cardiac abnormality.

Combination of GI and neurological symptoms are presentation of acute on chronic toxicity. These are most often precipitated by decreased glomerular filtration rate or intravascular volume depletion or intentional or unintentional over dose in patients on long term lithium ${ }^{1}$. Inadequate drug history may results in wrong clinical suspicion which can lead to wrong management. Long term use of lithium impairs secondary signaling pathway of dopamine mostly in limbic system and basal ganglia, as well as cerebellum resulting in neurological symptoms mimicking Parkinson's disease. Fine tremor with lower limb weakness without proper history may lead the physician to misdiagnose patient as a case of Parkinson's disease ${ }^{4}$.

The drug has a slow onset of action and it is neither protein bound nor metabolized and excreted unchanged through kidney which made it dialyzable. Lithium distribution from in and out of brain is slower and do not correlate with serum level. Neurological symptoms may persists even after dialysis due to redistribution of drug from $\mathrm{CNS}^{1}$. Neurological symptoms may consider irreversible if they persists for more than two months in the form of persistent cerebellar dysfunction, persistent extrapyramidal syndrome, persistent brainstem dysfunction, dementia, choreo athetoid movements, peripheral neuropathy (both motor and sensory), myopathy, optic neuritis etc. ${ }^{2,5}$.

Lithium level should be checked 12 hours after post ingestion (minimum 8 hours) of last dose to decide the management strategy. Sign and symptoms of lithium toxicity as well as lithium level guides the line of management. Gastro Intestinal decontamination, gastric lavage and activated charcoal are of limited use. Whole bowel irrigation with polyethylene glycol solution at $2 \mathrm{~L} / \mathrm{hr}$ is the only method of GI decontamination in case of acute poisoning provided there is no evidence of airway compromise or intestinal obstruction ${ }^{1}$. Adequate fluid resuscitation with maintenance of optimum sodium level and renal function contributes to a successful recovery. Avoidance of offending drugs that are known to increase lithium level (NSAID, ACE inhibitor, ARB, Thiazides, Spironolactone, diltiazem and verapamil) as well as avoidance of risk factors for chronic kidney disease are prerequisite for good outcome. In a population based cohort study by Clos $\mathrm{S}$ et al, stable lithium maintenance therapy does not increase the risk of renal dysfunction in adult patients with affective disorder. This study concludes that predictors for a decline in eGFR in patient receiving lithium, from the baseline eGFR are age,comorbidities, co prescription of nephrotoxic drugs and episodes of lithium toxicity, not the length of lithium exposure or mean lithium serum level ${ }^{6}$. In elderly patient and patient with chronic kidney disease low therapeutic range of lithium should be targeted ${ }^{7}$. Extracorporeal treatment( Dialysis) are recommended if serum lithium level is $>5 \mathrm{meq} / 1$ or $>4 \mathrm{meq} / 1$ with renal impairment or in presence of reduced level of consciousness, confusion, seizures or life threatening dysrhythmia irrespective of serum lithium level. ${ }^{8,9}$

Comprehensive history with patient cooperation may avert the misdiagnosis and erroneous treatment. A detailed patient and peer counseling and education regarding signs and symptoms of toxicity and importance of vigilant follow up before initiation of Lithium may help avoid the negative consequence. Patients who lives alone, atypical antipsychotics may be the alternatives of lithium in particular cases where Lithium therapy cannot be supervised.

\section{References:}

1. Mudan A, Dang H Jang. Toxicology In Tintinalli, editor, Tintinalli's Emergency Medicine, A comprehensive study guide, Ninth Edition. New york: McGraw Hill, 2020. p.1212-1214.

2. Schneider MA, Smith SS. Lithium-Induced Neurotoxicity: A Case Study. Journal of Neuroscience Nursing. 2019 Dec 1;51(6):283-6.

3. RM Steel, SM Lawrie. Medical Psychtry In Davidson's Principle and Practice of Medicine: Stuart H Ralston, Ian D Penman, Mark Wj Strachan, Richard P Hobson, editor, 23 ${ }^{\text {rd }}$ edition. Edinburgh: Elsevier, 2018. p. 1200. 
Bangladesh Crit Care J March 2021; 9 (1): 46-48

4. Aikoye SA, Parashar SD, Mann GS, Tinklepaugh M, CaseReport. Lithium Toxicity Misdiagnosed and Treated as Parkinson 's disease. Neurol Clin Therapeut J 2018, 2(1):1, p. 1-2.

5. Señga MM, Sarapuddin G, Saniel E. A Case Report on an Atypical Presentation of the Syndrome of Irreversible Lithium-Effectuated Neurotoxicity (SILENT) in a War Veteran with Bipolar Disorder and PTSD. Case reports in psychiatry. Jun 4;2020. doi: $\underline{10.1155 / 2020 / 5369297}$

6. Clos S, Rauchhaus P, Severn A, Cochrane L, Donnan PT. Long-term effect of lithium maintenance therapy on estimated glomerular filtration rate in patients with affective disorders: a population-based cohort study. The Lancet Psychiatry. 2015 Dec $1 ; 2(12): 1075-83$.
7. Foulser P, Abbasi Y, Mathilakath A, Nilforoo R, Do not treat the numbers: lithium toxicity BMJ Case Rep 2017. doi:10.1136/bcr-2017-220079,p1-3.

8. Okusa MD, Crystal LJ. Clinical manifestations and management of acute lithium intoxication. The American journal of medicine. 1994 Oct 1;97(4):383-9

9. De Cates AN, Morlet J, Reyad AA, Tadros G. Lithium overdose and delayed severe neurotoxicity: timing for renal replacement therapy and restarting of lithium. Case Reports. 2017 Oct 25;2017:bcr-2017 